\title{
MANAGEMENT AND ASSESSMENT OF HERITAGE BUILDINGS IN THE CITY OF HAVANA THROUGH THE PRIORITIZATION OF EMERGENCY ACTIONS
}

\author{
IGNACIO PIÑERO ${ }^{1}$, LEIRE GARMENDIA ${ }^{2}$, ALESANDRA GANDINI', JESÚS CUADRADO ${ }^{2}$, JOSÉ TOMÁS \\ SAN-JOSÉ ${ }^{3}$, IGNACIO MARCOS ${ }^{2} \&$ RAMÓN SANCIBRIÁN $^{4}$ \\ ${ }^{1}$ TECNALIA, Sustainable Construction, Spain. \\ ${ }^{2}$ UPV/EHU, Department of Mechanical Engineering, Spain. \\ ${ }^{3}$ UPV/EHU, Department of Mining, Metallurgical and Material Science, Spain. \\ ${ }^{4}$ University of Cantabria, Department of Structural and Mechanical Engineering, Spain.
}

\begin{abstract}
Declared a World Heritage Site by UNESCO, in 1982, the Historical Center of Havana, occupying an area of $2.14 \mathrm{~km}^{2}$, has 3,510 buildings, many of which now severely dilapidated. In fact, the Historical Center is in need of preferential action, due to the severity of the deterioration of its buildings. The situation is continually worsened following each hurricane season, and their accompanying tropical storms that have caused irreparable damage to buildings over the years. After a careful inspection of the heritage buildings of the Historical Center, it was possible to identify those in danger of imminent collapse and those with a useful life that could be prolonged through the implementation of the necessary emergency actions. In response to both challenges, a multi-criterion instrument based on the MIVES system was applied, with the purpose of prioritizing in an objective and justifiable way the necessary repairs and degrees of intervention. This instrument is based on requirements, criteria and indicators, all of which are selected, analysed and approved by a specially appointed panel of experts that quantifies all the factors in the value chain.
\end{abstract}

Keywords: building, historic heritage, MIVES, prioritization, rehabilitation

\section{INTRODUCTION}

The TECNALIA RESEARCH \& INNOVATION Foundation and the Office of the Historian of the City of Havana $(\mathrm{OHCH})$ have been collaborating together since 2002, through joint projects to manage the conservation of the Historical Centre of Havana, promoting the innovation, technology transfer and sustainable rehabilitation of Old Havana.

This project in particular is aimed at raising awareness among the local population for the preservation of the buildings and heritage, as well as training the technical staff of the $\mathrm{OHCH}$ in new rehabilitation technologies, providing them with a management tool for the prioritization of actions within the building stock of Old Havana.

The objective of this project emerged in response to the difficulties that the staff of the Master Plan for the Integral Revitalization of Old Havana (the planning body of the Office of the Historian) encountered with the prioritization of the repair work. There was an obvious need to develop a tool to support the prioritization of decision-making concerning emergency actions. A hierarchical scheme was first developed in which the different characteristics of the damaged buildings of the Historical Centre of Havana were described and classified, for the subsequent prioritization of the interventions in an objective and justifiable manner. The degree of each priority intervention was represented by an indicator based on the Integrated Value Model for Sustainability Assessment (MIVES Modelo Integral de Valor de Evaluación Sostenible), which resulted in an effective tool with which to plan the rehabilitation of the building stock. 


\section{THE CURRENT STATE OF THE BUILDINGS IN THE HISTORICAL CENTER OF HAVANA}

The Historical Center of Havana occupies an area of $2.1 \mathrm{~km}^{2}$, with 3,510 buildings, of which approximately 500 are considered very valuable. The remaining buildings in this built environment embody the architectural values that lend the city of Havana its distinctive character, reflecting a diversity of styles and historical periods that justify its status as a World Heritage Site.

The built environment of the Historical Center is characterized, to a large extent, by poor housing conditions, a quantitative and qualitative deficit of services, overcrowding and the subsequent deterioration of buildings destined for residential use (Fig. 1). The structural condition and façades of the buildings present alarming warning signs: $44 \%$ have structural pathologies in the roof, $42 \%$ show cracks or collapses in walls and façades, $24 \%$ show signs of structural collapses or failure of supporting members, $51 \%$ have water leaks through the roof or flooring and $38 \%$ leaks in walls; etc. [1].

The situation becomes even more serious, if we add the fact that many tenants are not owners and enjoy free usufruct (undoubtedly one of the most pressing problems of the Historical Centre) in an economy where the population lacks resources, building material suppliers and, in many cases, tenants lack the experience to carry out structural and even some minor repairs properly.

Moreover, the City of Havana is exposed to tropical storms and hurricane seasons, leaving the buildings of the Historical Centre, especially along the coastal fringe, vulnerable to extensive damage. In fact, according to an alarming statistic in Old Havana, collapses of diverse magnitude have regularly occurred every three days or so over several years [2].

Such a complex situation requires an entity that facilitates the coordination of all the stakeholders involved in the elaboration of an 'Integral Development Plan' that indicates the appropriate policies and strategies. It is worth noting here the remarkable work of the renowned historian of the city, Eusebio Leal Spengler, in charge of the Office of the Historian. In 1994, the Master Plan for the Integral Revitalization of the Historical Centre of Havana was launched and is currently headed by Patricia Rodríguez Alomá. It is responsible for coordination of the urban plan for the restoration and the rehabilitation of all buildings in the Historical Centre.

The technical inspectors of the Master Plan inspected all the buildings in the Historical Centre, thereby obtaining valuable updated information on their condition.
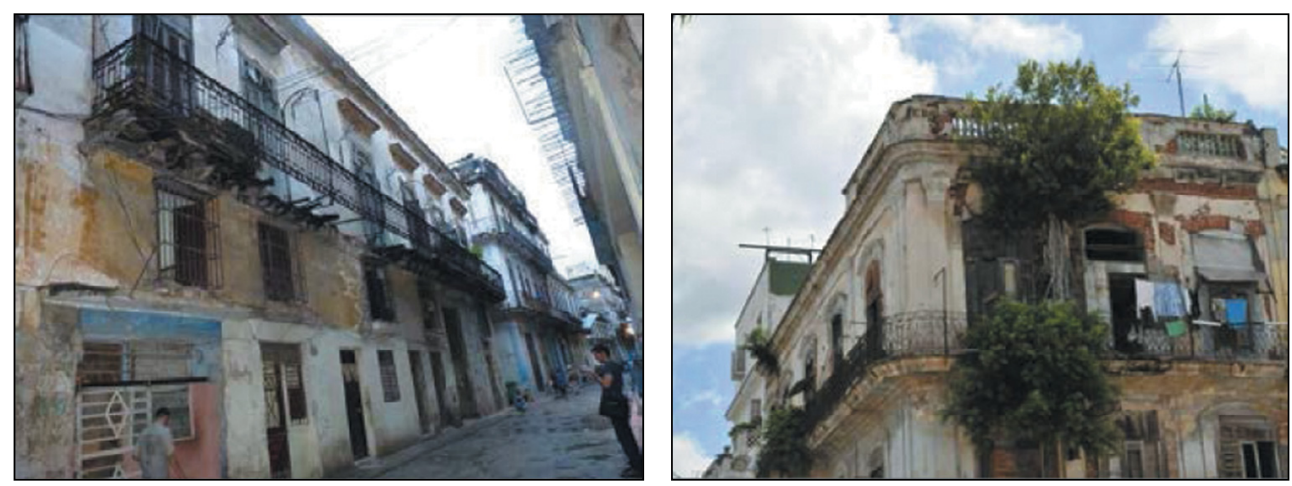

Figure 1: Dilapidated buildings in the Historical Center of Havana. 

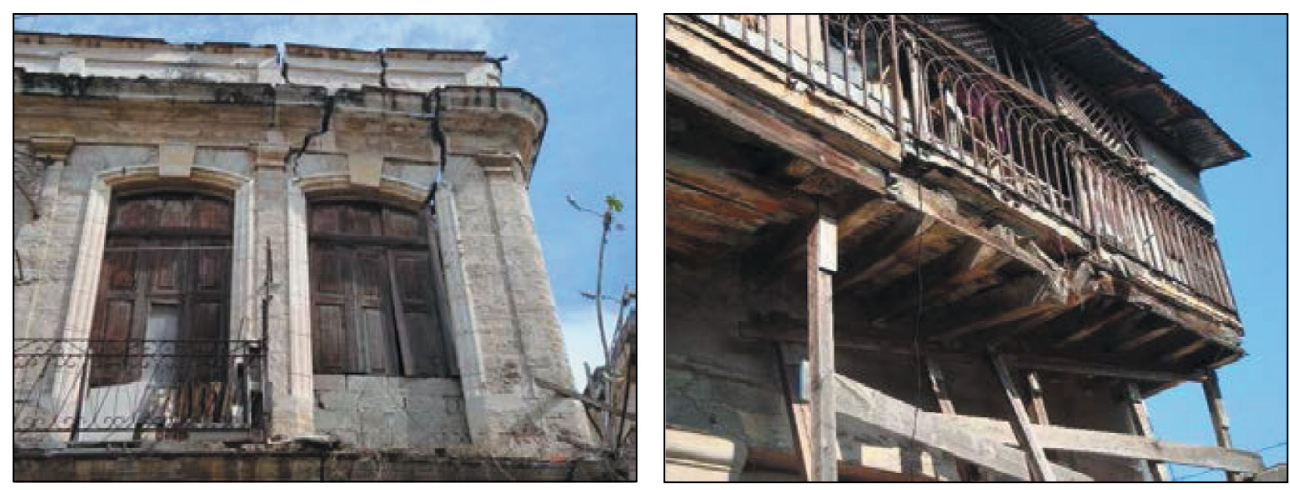

Figure 2: Need for emergency actions. A collapsing lintel (left) and balcony (right).

In all, 1,033 buildings were identified in the Historical Centre of Havana (out of the existing 3,510) in need of one or more emergency actions (representing $30 \%$ of the total) (Fig. 2). There were around 500 buildings (approximately 3,750 dwellings) in such a bad condition that it was not feasible to apply emergency actions, due to the degree of their deterioration, and around 200 buildings (1,300 houses approximately) that were in very poor condition. It was also found that around 1,700 buildings (approximately 14,350 dwellings) were in danger of some type of imminent collapse, placing the safety of their residents at risk, and/or with serious cracks that were deteriorating or could deteriorate their structures [3].

As already identified and developed by Rehabimed in other regions [4], the above-mentioned statistics highlight the need for an objective prioritization of the intervention strategy and an objective selection of the order for intervention on the buildings: the prioritization of emergency actions in the Historical Centre of Havana. Hence, the essential need for a tool to support the decision-making based on an objective and justifiable methodology for the interventions on the buildings in the Historical Centre of Havana.

\section{THE DEVELOPMENT OF A METHODOLOGY FOR THE MANAGEMENT OF BUILDING INFORMATION}

A methodology for the management of the information on the buildings was developed (see Fig. 3). Initially, a set of indicators to identify the technical characterization and structural condition of each building was determined. The following steps were followed in order to complete these indicators: (i) the definition of a systemic work program prior to the on-site inspection of the buildings, (ii) planning of the field work, (iii) office work to analyse and to process all the data and (iv) the drafting of a final report. At each stage, a checklist was prepared so that equivalent information was structured in all the cases under study. Additionally, it ensured that a systematic process was followed with a suitable monitoring system.

It should be noted that the resulting checklists were not intended to guide the structural diagnosis of the buildings. Instead, they served to record an organoleptic and primarily a visual inspection. A thorough diagnosis of the building was completed, once it had been selected for intervention.

The workflow (Fig. 3) allowed technicians to guarantee the quality of the work, avoiding carelessness, forgetfulness and irregularities between each intervention. The information gathered throughout the process was fed into the database on the current state of the building stock, thereby permitting the emergency actions needed for each building to be identified. 


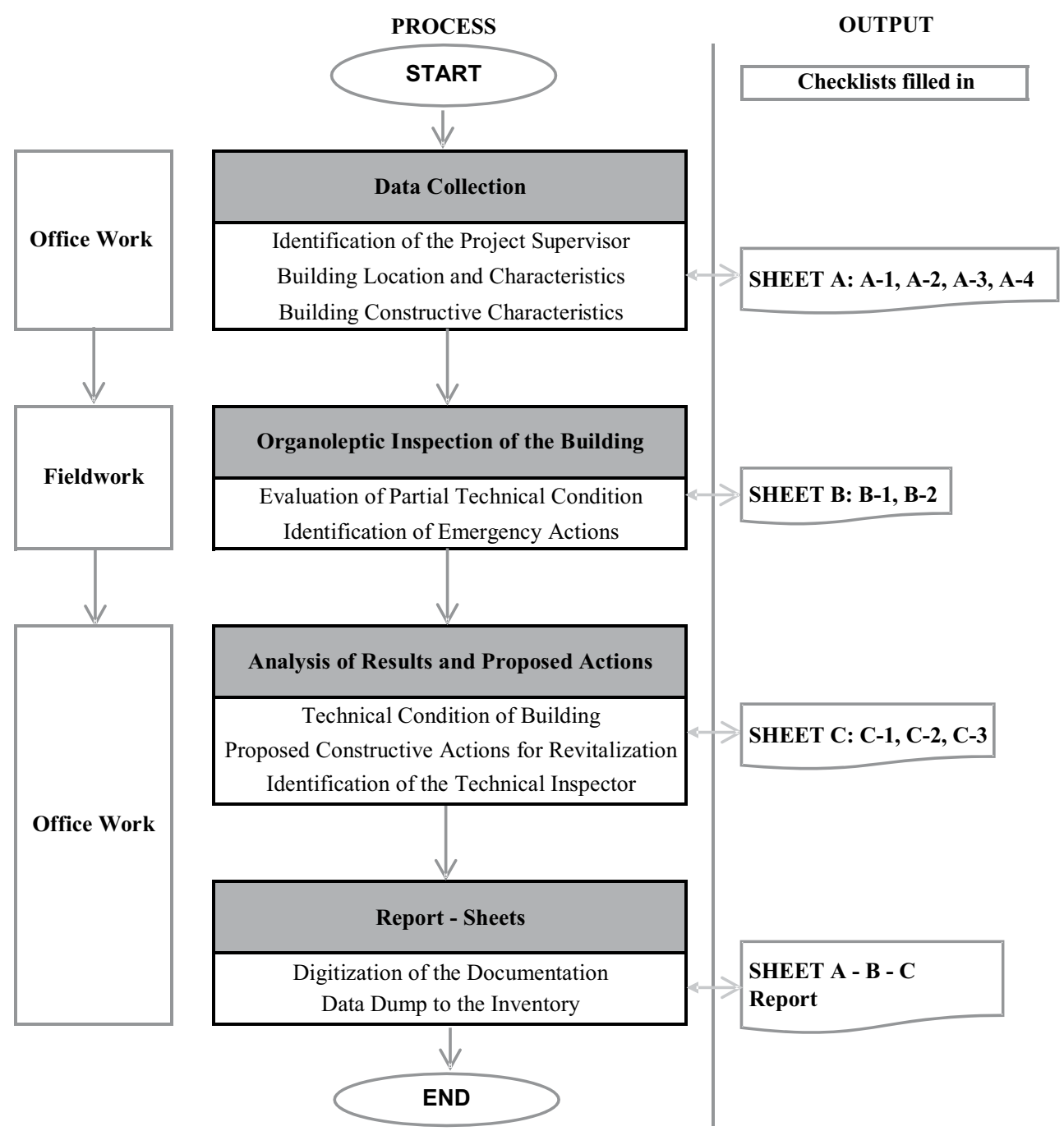

Figure 3: Workflow for systematic collection and analysis of the building information.

Once the inspection methodology had been agreed upon, an agreement between the Master Plan and the University of José María Echevarría (CUJAE) was signed, so that the students in the final year of their Civil Engineering degree could perform the inspections, as part of the 'Building Diagnostics' subject. The students used the checklists designed for that purpose.

The inspections were conducted on the buildings of the Historical Centre and the 'Prioritized Area for Conservation' that was extended to the Barrio Chino, in 2003, and to Malecón, along the coast line, in 2012 (both close to the neighbourhood of Centro Habana).

\section{PRIORITIZATION METHODOLOGY}

The basic concepts of the MIVES [5] methodology (Fig. 4) were applied to define the order of prioritization. Additionally, so as to understand the rehabilitation work carried out in Havana, as the city historian Eusebio Leal remarked [6], 'you have to travel through the history of 


\begin{tabular}{|l|l|l|}
\hline Block I: DEFINITION OF MISSION & Block II: ENVISIONED SOLUTION & \multicolumn{1}{|c|}{ Block III: DECISION-MAKING } \\
\hline$\rightarrow$ Problem definition & $\rightarrow$ Relative weights allocation & $\rightarrow$ Results obtained \\
$\rightarrow$ Value Functions definition & $\rightarrow$ Valuation of alternatives & $\rightarrow$ Results dissertation \\
& $\rightarrow$ Computer tool implementation & $\rightarrow$ Timely decision-making \\
\hline
\end{tabular}

Figure 4: MIVES methodological scheme.

the Historic Centre'. Therefore, during the application of the methodology, there was direct collaboration with the personnel in the Office of the Historian who helped to explain the state of conservation of the building stock and to implement the aforementioned objective of prioritization.

A methodology based on the set of previously defined indicators was proposed. This methodology, on the one hand, identifies the constructive actions that the buildings require and, on the other hand, prioritizes the degree of intervention that is needed.

The use of a methodology that takes into account the most varied aspects of the problem (decision domains) and their hierarchy is fundamental. The methodology must be as objective as possible, so that all the data may be compiled and processed, in order to establish the prioritization of the rehabilitation intervention. The methodology can validly be applied to a large number of buildings in poor condition with varied qualifications (from the least bad to the worst condition and without forgetting that it is an environment declared as World Heritage).

MIVES is made of two basic aspects [7]. Firstly, the establishment of a requirement tree composed of criteria/sub-criteria/indicators [8], in which relative values (weights) are defined using pairwise comparison, following the Analytic Hierarchy Process established by Saaty in 1971 [9]. Secondly, the basic units of the tree (indicators) are normalized to a dimensionless and standardized 'value' through value functions [10].

As a consequence of the characteristics of the decision-making process, the use of MIVES methodology can justify a single value index, called the Prioritization Index. This index is a summary of the ratings given to factors considered in the criteria/subcriteria/indicators that established not only the required intervention needs of the buildings in the top positions but also the hierarchy of all the buildings. There is also the possibility of assessing other alternatives in the future, without altering the previous evaluations [11].

A panel of experts was appointed, in order to establish the values for the requirements tree, which assists the management of the Master Plan in its decision-making. The panel included staff specialized in technical, sociological and patrimonial issues and was appointed with the help of the TECNALIA Foundation and the University of the Basque Country (UPV/EHU). It was under the constant supervision, and it functioned with the collaboration of the staff of the Office of the Historian of Havana, all with equivalent professional profiles.

Subsequently, a requirements tree, based on 19 unique and acceptable indicators for the specific case of the inspection and the rehabilitation of the Historical Centre of Havana, was constructed. Finally, the information collected, which can be updated when necessary, together with the sheets to obtain real data of the buildings during the fieldworks was validated. And, from here on, the evaluation strategy was implemented, which transformed the value functions, relating to qualitative or quantitative aspects, into a dimensionless variable of between 0 and 1 . 


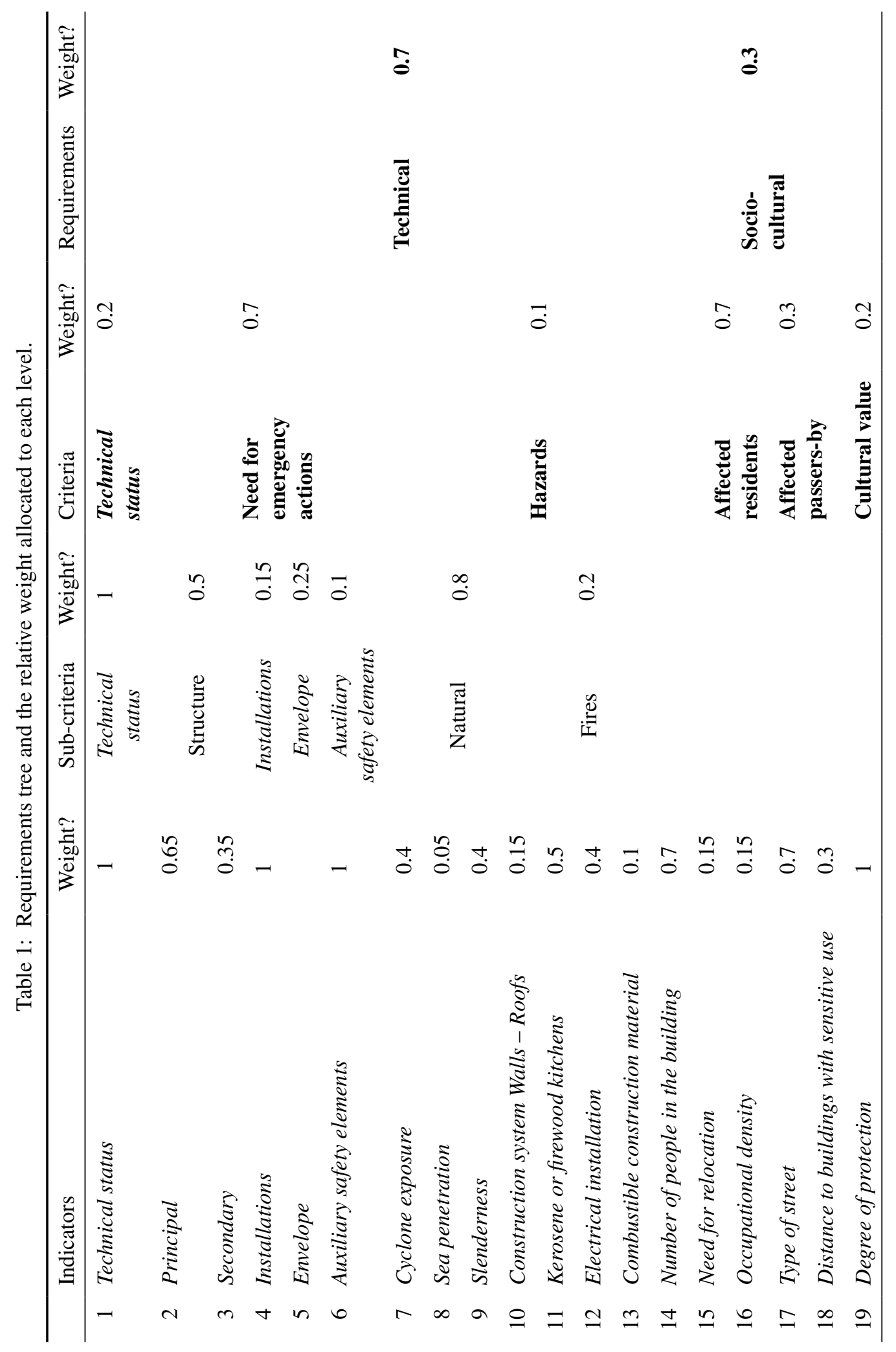


The panel of experts with the purpose of rehabilitating the buildings of Havana defined, compared and agreed upon 19 indicators (see Table 1). The technical and socio-cultural requirements defined in the mission were sufficient to tackle a problem as complex as the rehabilitation of a complete urban area. For this purpose, weights were assigned to the different levels of the requirements tree according to their relative importance. All the levels were assessed: the smallest and most specific (Indicator), the complete units of study (Criterion) and, finally, the global scale (understood as unified fields of study called Requirements).

When the requirements tree had been defined, the relative weights were allocated, the evaluation of all indicators was performed and a relevant sensitivity analysis was performed, validating the feasibility and effectiveness of the proposed methodology. The final value, between 0 and 1 , called the sustainable priority index (SPI), guided the prioritization of the interventions on the buildings.

\section{MANAGEMENT SOFTWARE TOOL BASED ON THE PRIORITIZATION METHODOLOGY}

Once the requirements tree had been designed and assigned values, the software tool was developed. The software integrated the quantification methodology into an application which, based on a workflow, provided the prioritization list of the buildings of the Historical Centre of Havana that require emergency actions, which was the main aspect and the key objective of the FOCAD mission [12].

Likewise, the software tool was also designed with the objective of developing an application that could provide information on the Master Plan Web page (www.planmaestro.ohc. cu). Related information may be consulted on the same website (www.planmaestro.ohc.cu/ app/emergencia/).

Having completed the inspections and collected the information, the script was compiled using the PHP language. The resulting list can be requested from the Office of the Historian that keeps records of all the available information that is processed.

The positions of the buildings in the ranking were verified, in order to validate the results of the management software tool. This validation was done by means of inspection of a large number of the buildings. Moreover, photographs taken during the last inspection were also consulted to compare and to verify the relative degrees of deterioration.

\section{DISCUSSION OF RESULTS}

Figure 5 presents the constructive actions identified in the project. The results show that the buildings located in the South (San Isidro and Belén districts) included a higher concentration of buildings that required emergency actions (shown in brown in the figure). This observation is due to the fact that the Historical Centre expanded from the South to the North. Therefore, the oldest buildings are located in the South and are characterized by poor constructive systems and overcrowded areas.

Once the parameters were introduced in the computer software, 1,033 buildings in the Historical Centre of Havana were identified as requiring one or more emergency actions. The SPI was used, in order to prioritize the interventions among these buildings. The SPI values for the building stock follow a sinusoidal curve with continuous values of between 0.7 and 0.1 . Hence, the building stock was divided into four groups (Table 2) depending on the SPI, in order to prioritize the intervention needs of the buildings. In this way, the efforts were focused on rehabilitation of the buildings at the top of the list. 


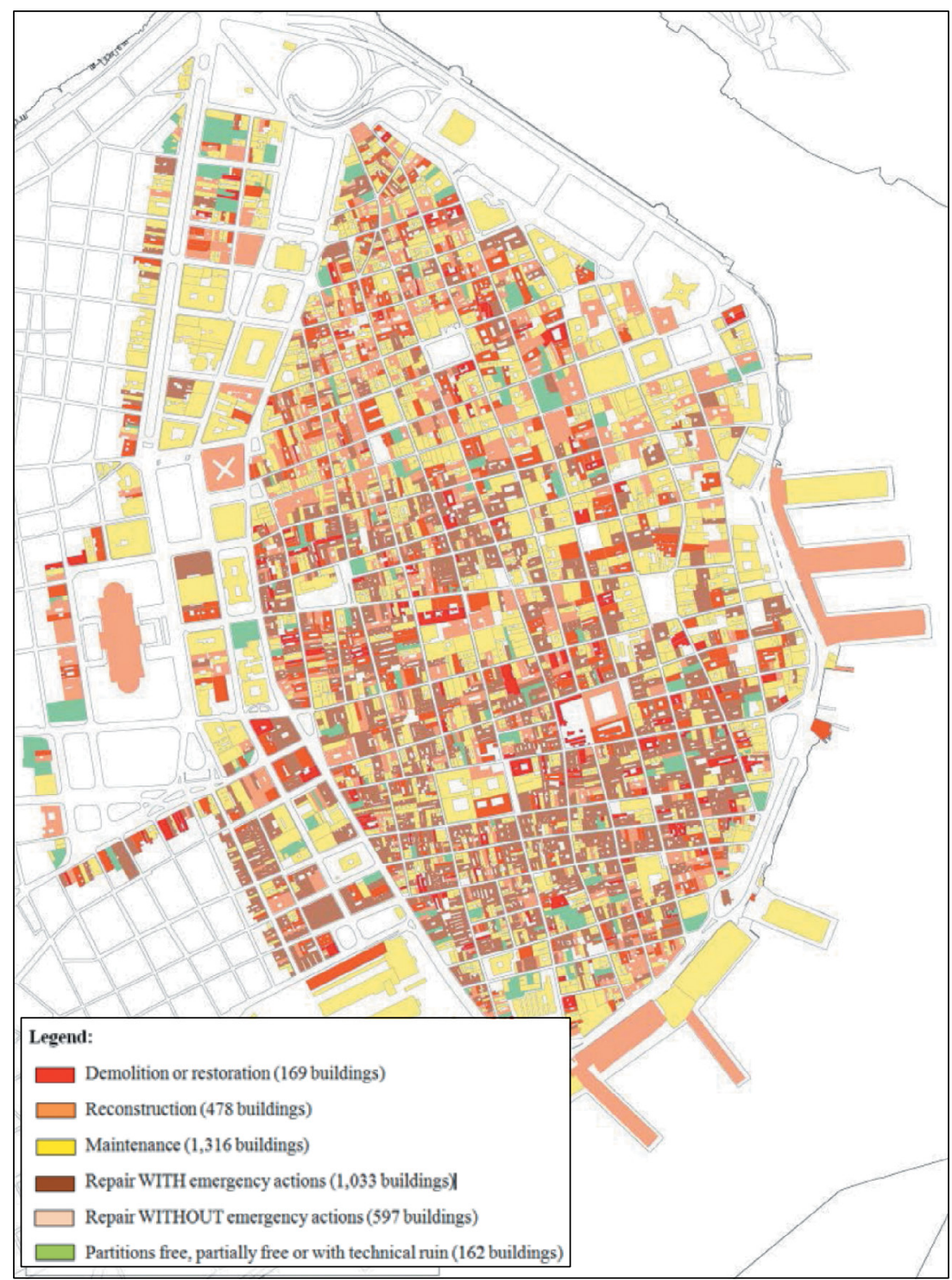

Figure 5: Identification of constructive actions.

The methodology allowed technicians to identify the constructive action needed in the study area that was foreseen in the plans of the Master Plan Office, according to several aspects that had been verified. First, they were in absolute agreement with the buildings marked as rehabilitated and with the buildings that only required maintenance works. Secondly, they also agreed with the list of buildings that were classified as 'in ruins'. And thirdly, several buildings that they had already marked as awaiting large-scale intervention work matched the results. Hence, the prioritization confirmed and improved the knowledge of the experts of the rehabilitation plan of Havana. It has allowed them to prioritize the building stock of the area and to plan their interventions in a more conscious, precise and effective way. 
Table 2: Different priority intervals for buildings requiring emergency action.

\begin{tabular}{lll}
\hline Priority & Range SPI & Number of buildings \\
\hline High & $1-0.57$ & 56 \\
Medium-high & $0.57-0.41$ & 338 \\
Medium-low & $0.41-0.26$ & 453 \\
Low & $0.26-0$ & 186 \\
Total & $1-0$ & 1,033 \\
\hline
\end{tabular}

It must be highlighted that the tool provides a list of buildings and that the first one is not necessarily the most suitable for rehabilitation. The tool provides a list of buildings that will support the decisions over which buildings should be considered for intervention, justifying their prioritization and providing reasons for a proper choice. It is not an automatic process and is never a replacement for the on-site judgment of qualified experts.

The versatility of the tool was demonstrated by means of exporting the list to a spreadsheet to produce filters, graphics etc. so that the Master Plan management team could use the information to take the proper decision more easily and effectively.

\section{CONCLUSIONS}

This project has made it possible to develop a methodology for the management of building information in a systematic way. A process involving both field and office work has been developed, and specific checklists are used to gather information for processing. Additionally, a prioritization tool based on MIVES methodology has been developed. The tool has allowed the experts to establish a list of buildings based on the index of sustainable prioritization for renovation purposes, identifying necessary constructive actions to maintain the building stock of the Historical Centre of Havana and the 'Prioritized Area for Conservation'.

Furthermore, the results of the management software tool and the display of the geo-referenced plans have demonstrated that the results matched the spatial intervention strategy previously developed by the Master Plan technicians.

\section{ACKNOWLEDGEMENTS}

The authors appreciate the participation of the Research Group formed by the UPV/EHU and TECNALIA under the agreement IT781-13 with the Basque Government. They also wish to express gratitude to the MIVES research group for its support in the use of the methodology, and to Patricia Rodríguez Alomá, Pablo Fornet, Raimundo de la Cruz and Juan Carlos Bresó for their assistance in this project. Finally, the authors would like to extend their gratitude to all those who have collaborated in the expert decision-making panel for their dedication and participation, freely sharing their experience and good judgement.

\section{REFERENCES}

[1] Plan Maestro. Special plan for integral development. Office of the Historian of the City of Havana, Office of the Historian of the City of Havana, Havana, Cuba, 2011 (in Spanish).

[2] Mutal, S. \& Carrión, F. A unique experience. Valuations on the integral management model of Old Havana, World Heritage Site. UNESCO - Office of the Historian of the City of Havana, Ed. Boloña, Havana, Cuba 2006 (in Spanish). 
[3] De la Cruz Luzardo, R., Instructive. State technical inventory. constructive systems and emergency actions. Master Plan for the Integral Revitalization of Old Havana - Office of the Historian of the City of Havana, Havana, Cuba, 2011 (in Spanish).

[4] Rehabimed Association, available at http://www.rehabimed.net/. Accessed on 3 May, 2017.

[5] MIVES. Integrated model for quantifying the value of a sustainable construction project. Application to the industrial building and services. MAT2002-04310-C03-02, L Ministerio de Ciencia y Educacion, (in Spanish).

[6] Leal Splenguer, E., The rehabilitation of the Historic Center of Havana: An essentially human work. Conference Held in the Framework of the Debate "Urban Traumas: City and Disasters”. CCCB, 7-11 July 2004 Universal Forum of Cultures. Barcelona, 2004 (in Spanish).

[7] Reyes, J.P., San-José, J.T., Cuadrado, J. \& Sancibrián, R., Health \& safety criteria for determining the sustainable value of construction projects. Safety Science, 62, pp. 221232, 2014. DOI: 10.1016/j.ssci.2013.08.023

[8] San-José, J.T. \& Garrucho, I. A system approach to the environmental analysis of industrial buildings. Building and Environment, 45(3), pp. 673-683, 2010. https://doi. org/10.1016/j.buildenv.2009.08.012.

[9] Saaty, TL., Decision making with the analytic hierarchy process. International Journal of Services Sciences, 1(1), pp. 83-98, 2008. DOI: 10.1504/IJSSCI.2008.017590

[10] Alarcón, B., Aguado, A., Manga, R. \& Josa, A. A value function for assessing sustainability: Application to industrial buildings. Sustainability, 3(1), pp. 35-50, 2011. DOI: 10.3390/su3010035.

[11] Piñero Santiago, I., Methodology to Prioritize and Plan, in a Sustainable Way, the Rehabilitation of Degraded Structures. Extreme Case of the Historic Center of Havana, $\mathrm{PhD}$ thesis. University of the Basque Country, Bilbao, Spain, 2013 (in Spanish).

[12] TECNALIA - Special Plan for Integral Development. Conservation actions for the habitability of the architectural heritage of Old Havana. Fund for Development Cooperation and Assistance (FOCAD) of the Basque Government (PRO-2010K2/0001). 